\title{
Gestión del presupuesto por resultados para mejorar la calidad del gasto público en la unidad de gestión educativa local San Martín - Tarapoto
}

\author{
Elaine Dávila Rodríguez \\ elaine_dr@hotmail.com
}

Dr. Keller Sánchez Dávila
ksanchezd@ ucv.edu.pe
Universidad César Vallejo

\section{RESUMEN}

La investigación se planteó con el objetivo de establecer la gestión del presupuesto por resultado para mejorar la calidad del gasto público en la unidad de Gestión educativa Local UGEL San Martín; dentro del el propósito se buscó conocer las características de la gestión por resultado y como este incide en la mejora de la calidad del gasto. Proponiendo soluciones mediante las conclusiones. La investigación fue descriptiva simple utilizando revisiones sistemáticas de estudios para establecer resultados, la mismas que se realizaron a nivel internacional y nacional referente a las variables. Entre los resultados la gestión del presupuesto por resultado promueve una gestión educativa eficiente y descentralizada con enfoque participativo de la sociedad, familias, ciudadanos y ciudadanas para la implementación de políticas educativas basadas en la gestión por resultados con enfoque a la comunidad de aprendizaje profesional y a la prestación de servicios educativos. Como el desarrollo profesional del maestro, el fortalecimiento de mecanismos de garantía que asegure la cantidad suficiente de materiales educativos. La calidad del gasto en educación no garantiza que los niños reciban los recursos que demandan para obtener la calidad de los aprendizajes ya que ellos necesitan alimentos, vivienda y buena salud. El sector educativo señala que la práctica educativa añade valor a la eficiencia del gasto público y promueve las inversiones en las escuelas públicas, el desempeño educativo juega una labor importante en el desarrollo de los niños originando la igualdad de oportunidades de aprendizaje a largo del ciclo presupuestario. Concluyendo que la gestión del presupuesto por resultado permite mejorar las condiciones institucionales, principalmente incorporando el 
bienestar público, la eficacia y competitividad del gobierno, en las políticas educativas. La calidad del gasto público optimiza y da solución a problemática existente.

Palabras Clave: gestión del presupuesto por resultado; calidad del gasto público; bienestar público; eficacia, competitividad del gobierno; participación; políticas educativas. 


\title{
Management of the budget by results to improve the quality of public spending in the local educational management unit San Martín - Tarapoto
}

\begin{abstract}
The research was proposed with the objective of establishing the management of the budget by result to improve the quality of public spending in the Local Educational Management unit UGEL San Martín; Within the purpose, it was sought to know the characteristics of management by result and how this affects the improvement of the quality of spending. proposing solutions through conclusions. The research was simple descriptive using systematic reviews of studies to establish results, the same ones that were carried out at the international and national level regarding the variables. Among the results, the management of the budget by result promotes efficient and decentralized educational management with a participatory approach of society, families, citizens and citizens for the implementation of educational policies based on management by results with a focus on the professional learning community and the provision of educational services. Like the professional development of the teacher, the strengthening of guarantee mechanisms that ensure a sufficient quantity of educational materials. The quality of spending on education does not guarantee that children receive the resources they demand to obtain quality learning since they need food, shelter and good health. The education sector indicates that educational practice adds value to the efficiency of public spending and promotes investments in public schools, educational performance plays an important role in the development of children, causing equal learning opportunities throughout the budget cycle. Concluding that the management of the budget by result allows to improve the institutional conditions, mainly incorporating the public welfare, the efficiency and competitiveness of the government, in the educational policies. The quality of public spending optimizes and solves existing problems.
\end{abstract}

Keywords: budget management by result; quality of public spending; public welfare; efficiency; government competitiveness; participation; education policies.

Artículo recibido: 18. Julio. 2021 Aceptado para publicación: 08. Agosto. 2021 Correspondencia: elaine dr@hotmail.com Conflictos de Interés: Ninguna que declarar 


\section{INTRODUCCIÓN}

En la actualidad las entidades públicas son encargadas de tomar medidas necesarias para el cumplir las metas programadas reduciendo las brechas de la educación pública y la privada, la educación rural y la urbana, siendo indispensable la educación de calidad para todos los ciudadanos y ciudadanas sin exclusiones, priorizando e impulsando la educación en la primera infancia, el uso de nuevas tecnologías educativas, permitirá la acreditación de las Instituciones que ofrecen el servicio educativo, ofreciendo oportunidades de educación y el desempeño profesional docente, impulsando el compromiso intersectorial, eficiente y descentralizada, altamente profesional con principios de ética pública, y amplia participación de la sociedad, principalmente de las familias. Según, Batista Rodríguez, Leyva Figueredo , \& Mendoza Tauler (2021), menciona que históricamente la educación es la expresión social definido, como eje del desarrollo socializador que establece una atribución definitiva en el desarrollo del individuo durante su vida, y debe prepáralo tanto para el logro de una formación personal y social activa, como para el goce y plenitud.

\section{Teorías de las variables}

\section{Gestión del Presupuesto por resultado}

La gestión del presupuesto por resultado, se relaciona con la implementación de políticas educativas, ya que supervisan las acciones de rendición de cuentas que sirven para identificar las medidas requeridas donde se fomenta la "gestión basada en resultados" (GBR), el enfoque de la comunidad de aprendizaje profesional, asegura esta responsabilidad al permitir que los miembros recopilen, gestionen y analicen los datos de los estudiantes. La gestión basada en resultados (GBR) propone dos series de actividades docentes relacionadas con la priorización de tareas y el papel de la comunidad de aprendizaje profesional en un contexto de intensificación del trabajo y gestión basada en resultados (GBR) Bouchamma \& April (2020).

Por otra parte, Dev Bhatta, Rahman, Rahman, Sharma, \& Adms (2020) sostiene que el número de niños matriculados en programas de Evaluación Censal de Estudiantes (ECE) aumente sustancialmente en los próximos años, lo que requerirá mayores recursos financieros y capacidad de prestación de servicios; requiriendo actualmente mayores niveles de inversiones en obras de impacto social como el perfeccionamiento profesional de los maestros, el fortalecimiento de la competitividad en las instituciones 
de formación de maestros, el fortalecimiento del mecanismo de garantía de calidad y la provisión de un número suficiente de aulas para el escuelas primarias públicas

\section{Calidad del Gasto Publico}

La calidad del gasto público, señala que el desempeño educativo a través de la calidad del gasto del sector educativo mejora la función de supervisión del plan de desarrollo de persona a lo largo del ciclo presupuestario añade valor a la calidad del gasto del sector educativo y puede fomentar simultáneamente un mejor desempeño educativo Yatun, Mulyani, Winarningsih, \& Sukmadilaga (2021).

Por otro lado, Marin, Acosta Roa, \& Arriojas Tocuyo (2020) sostiene que la ejecución del gasto en educación en el Perú es más baja en el mundo y se observa que va en aumento, no se tiene eficiencia, lo que evidencia desigualdades en educación de calidad, reduciendo la tasa de matrícula y el retraso escolar que produce repitencia y deserción.

Asimismo, se ha evidenciado que en la Unidad de Gestión Educativa Local UGEL San Martín existe situaciones problemáticas en la asignación de recursos financieros y el uso eficiente del mismo con objetivos educativos que demanda la población. Existen capacidades limitadas en la implementación de actividades que se articulen con los programas presupuestales. También es importante mencionar la calidad del gasto en todas las compras se realizan sin evaluar la calidad del producto por el cual fue presupuestado, probablemente no se está desarrollando debidamente la ejecución presupuestal de la institución, lo que ha ocasionado el descontento de los beneficiarios y usuarios, no se está entregando una atención de calidad. Este contexto está atentando contra la calidad educativa pues no se está considerando los intereses de los estudiantes. Por lo tanto, la situación problemática no permite mejorar el presupuesto existente y manejarlo adecuadamente en un eficiente gasto que esté de acuerdo a lo planificado evitando las recargadas comisiones de compras o hasta la corrupción de los colaboradores de la Unidad de Gestión Educativa Loca - UGEL San Martín.

Por lo mencionado, se ha determinado realizar la presente investigación, debido que a la actualidad esta institución debe optimizar el gasto público ya que existe muchas necesidades por atender, es por esta razón se plantea poner en práctica la gestión del presupuesto por resultados como un medio para solucionar el problema existente y dar como una propuesta para mejorar la calidad del gasto público en la Unidad de Gestión educativa Local - UGEL San Martín. 


\section{Formulación del Problema}

De acuerdo con la problemática descrita, se propone como problema general:

- ¿De qué manera la gestión del presupuesto por resultados contribuye a mejorar la calidad del gasto público en la unidad de Gestión Educativa Local San Martín Tarapoto?

\section{Problema específico}

1) ¿Cómo es la gestión del presupuesto por resultado en la Unidad de Gestión Educativa Local San Martín - Tarapoto?;

2) ¿Cómo es la formulación de estrategias de la gestión presupuesto por resultado en la Unidad de Gestión Educativa Local San Martín - Tarapoto?;

3) ¿Cuál es la técnica de elaboración de la gestión del presupuesto por resultado en la Unidad de Gestión Educativa Local San Martin - Tarapoto?

\section{MATERIAL Y MÉTODOS}

\section{Diseño de estudio}

En la investigación no recurrió a la manipulación de variables es de tipo no experimental, descriptivo, describiendo las variables de administración financiera y desarrollo local mediante las revisiones bibliográficas de revistas. Tiene enfoque cualitativo, recurriendo a la revisión sistemática de investigaciones científicas de revistas indexadas de los últimos 2 años, constituyéndose como unidades de análisis con información original y primaria. Los mismo son instrumentos fundamentales para el análisis y síntesis de la información encontrada, identificando los principales problemas válidos para las conclusiones del estudio.

La técnica fue la revisión bibliográfica y el instrumento la guía de análisis mediante tablas respectivas. Los materiales utilizados fueron tablas de análisis de bibliografía especializada referente a las variables de estudio, usando el Excel y Word, incluyendo laptop. Respecto al método se utilizó el hipotético deductivo, partiendo de particularidades con información internacional y nacional, para llegar a hechos generales para llegar a conclusiones generales

\section{Procedimiento de recolección de datos}

El proceso de recolección se dio mediante la selección de información de acuerdo a la temática de investigación de los últimos 2 años de revistas indexadas, siempre y cuando se tuvo acceso al texto completo de los mismos para el análisis respectivo. La 
recolección de información con bibliografía especializada se consideró las tablas de análisis se tuvo en cuenta información general donde se ubica nombre del autor, título, año, ubicación de revista y lugar, en la parte de desarrollo va la metodología, principales resultados y conclusiones, y el análisis al final. Se incluyó todas las investigaciones con información relevante y se excluyó aquellas con menos relevancia para la investigación. Técnicas de análisis de datos. La técnica de análisis de datos se dio mediante la revisión sistemática por comparación de las características de las variables, teniendo en cuenta las similitudes y discrepancias entre las investigaciones de artículos científicos a nivel internacional y nacional. Acto seguido, se realizaron las evaluaciones críticas de cada artículo mediante la discusión de resultados, siendo el principal insumo para las conclusiones respectivas.

Referente a la parte ética, se utilizó estándares internacionales de transparencia y justicia, citando a los autores utilizados mediante el estilo APA, con los aportes respectivos de cada uno de ellos.

\section{RESULTADOS.}

Los resultados se realizaron mediante tablas de análisis, teniendo en cuenta artículos de revistas indexadas a nivel internacional y nacional referente a las variables de estudio de la siguiente forma: 
Tabla 1

Datos de autor 1

\begin{tabular}{|c|c|c|c|c|}
\hline 1. Autor & Año & Título & Link & Revista \\
\hline
\end{tabular}

\section{DESARROLLO}

\begin{tabular}{|c|c|c|c|c|c|}
\hline Metodología & Muestra & Instrumentos & Ética & Resultados & Conclusiones \\
\hline $\begin{array}{l}\text { Básico } \\
\text { Descriptivo }\end{array}$ & 53 Hogares & $\begin{array}{l}\text { Se aplicó el } \\
\text { Cuestionario }\end{array}$ & $\begin{array}{l}\text { Ética } \\
\text { institucional }\end{array}$ & $\begin{array}{l}\text { La educación en la primera infancia } \\
\text { escuelas primarias públicas es } \\
\text { fundamental para mejorar los } \\
\text { resultados del desarrollo infantil y } \\
\text { garantizar que la próxima generación } \\
\text { de niños de Bangladesh esté mejor } \\
\text { preparada para la escuela. Bangladesh } \\
\text { ha logrado un progreso notable en el } \\
\text { aumento de la accesibilidad en la } \\
\text { educación escolar y asegurar que más } \\
\text { niños completen la escuela. Sin } \\
\text { embargo, se estima que, si bien los } \\
\text { niños de Bangladesh completan un } \\
\text { promedio de } 11 \text { años de escolaridad, } \\
\text { sus años de escolaridad ajustados al } \\
\text { aprendizaje son de solo } 6,5 \text { años } \\
\text { (Banco Mundial 2018). }\end{array}$ & $\begin{array}{l}\text { El número de niños matriculados en programas de } \\
\text { Evaluación Censal de Estudiantes (ECE) aumente } \\
\text { sustancialmente en los próximos años, lo que } \\
\text { requerirá mayores recursos financieros y } \\
\text { capacidad de prestación de servicios; requiriendo } \\
\text { actualmente mayores niveles de inversiones en } \\
\text { obras de impacto social como el } \\
\text { perfeccionamiento profesional de los maestros, el } \\
\text { fortalecimiento de la competitividad en las } \\
\text { instituciones de formación de maestros, el } \\
\text { fortalecimiento del mecanismo de garantía de } \\
\text { calidad y la provisión de un número suficiente de } \\
\text { aulas para el Escuelas primarias públicas. }\end{array}$ \\
\hline
\end{tabular}

Análisis. El análisis muestra que le estado tiene que invertir en mejorar la calidad en educación, como el desarrollo profesional de los docentes, el fortalecimiento de capacidades y la provisión de números de aulas.

Ciencia Latina Revista Científica Multidisciplinar, Ciudad de México, México.

ISN 2707-2207 / ISSN 2707-2215 (en línea), julio-agosto, 2021, Volumen 5, Número 4 https://doi.org/10.37811/cl_rem.v5i4.769 p.6255 
Tabla 2

Datos de autor 2

\begin{tabular}{ccllll}
\hline 2. Autor & Año & Título & Link & Revista \\
\hline Bouchamma, Yamina; & 2020 & La comunidad de aprendizaje & Administración & y & Política \\
April, Daniel & & profesional para implementar & https://files.eric.ed.gov/fulltext/EJ1252284.pdf & Educativa \\
& & el Enfoque de gestión basada & Canadá & vol. 192 \\
& en resultados (GBR) en & & \\
& Quebec & &
\end{tabular}

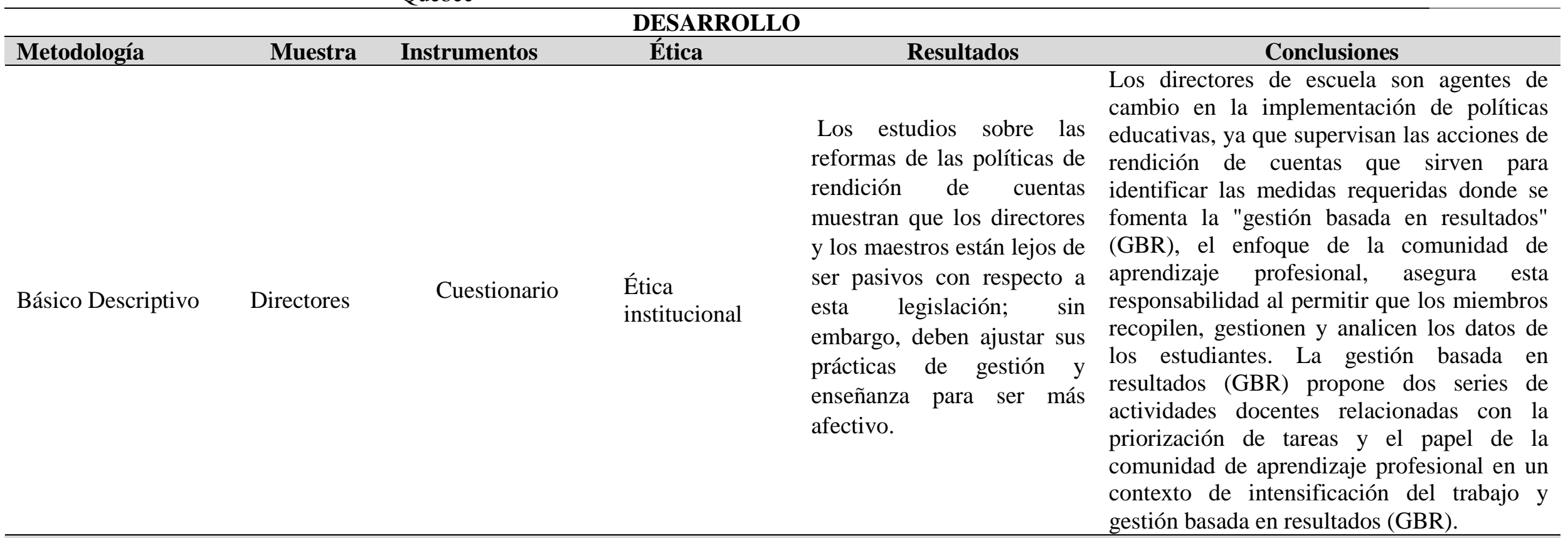

Análisis: Es posible examinar la gestión basada en los resultados en las escuelas con políticas de rendición de cuenta utilizadas por los líderes escolares para desarrollar tanto la gestión de la gestión pedagógica como una herramienta para implementar la estructura operativa de la institución. 


\section{Tabla 3}

Datos de autor 3

\begin{tabular}{|c|c|c|c|c|}
\hline 3. Autor. & Ã̃o & Título & Ubicación de la revista & Volumen y número. \\
\hline $\begin{array}{l}\text { Batista-Rodríguez, Luis } \\
\text { Felipe; Leyva-Figueredo, } \\
\text { Prudencio Alberto; } \\
\text { Mendoza-Tauler, Laura } \\
\text { Leticia }\end{array}$ & 2021 & $\begin{array}{l}\text { Gestión y desarrollo de la formación } \\
\text { laboral en la educación actual }\end{array}$ & $\begin{array}{l}\text { https://www.redalyc.org/articulo.oa?id=1815657 } \\
09003 \\
\text { Cuba }\end{array}$ & $\begin{array}{l}\text { Ciencias Holguín } \\
\text { vol. } 27 \text {, núm. } 1\end{array}$ \\
\hline
\end{tabular}

Leticia

\section{CUERPO}

\begin{tabular}{|c|c|c|c|c|c|}
\hline \multicolumn{6}{|c|}{ CUnin } \\
\hline Metodología & Muestra & Instrumentos & Ética & Resultados & Conclusiones \\
\hline $\begin{array}{l}\text { Básico } \\
\text { Descriptivo }\end{array}$ & $\begin{array}{l}\text { Trabajador } \\
\text { es } \\
\text { egresados } \\
\text { de la } \\
\text { universidad }\end{array}$ & Cuestionario & $\begin{array}{l}\text { Ética } \\
\text { institucional }\end{array}$ & $\begin{array}{l}\text { El estudio tiene como resultado que } \\
\text { existe insuficiente desempeño } \\
\text { profesional de colaboradores, } \\
\text { motivo por la cual se formuló } \\
\text { estrategias de solución basa en la } \\
\text { gestión de formación profesional y } \\
\text { laboral que expliquen los elementos } \\
\text { para el desarrollo profesional y un } \\
\text { mejor desempeño. }\end{array}$ & $\begin{array}{l}\text { La educación es expresión social } \\
\text { históricamente definido, como eje del } \\
\text { proceso socializador que ejerce una } \\
\text { atribución decisiva en la formación del } \\
\text { individuo a lo largo de toda su vida, y debe } \\
\text { prepáralo tanto para el logro de una } \\
\text { incorporación personal y social activa, como } \\
\text { para el goce y plenitud. }\end{array}$ \\
\hline
\end{tabular}

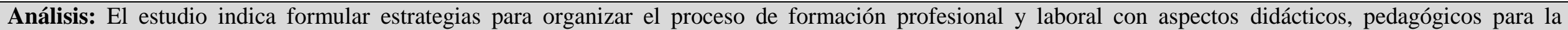
formación del individuo. 


\section{Tabla 04}

Datos de publicación de autor 04.

4. Autor.

\section{Año}

Monterubbianesi, Pablo Daniel;

Rojas, Mara Leticia; Dabús, 2021 Carlos Darío $\begin{array}{lc}\text { Educación y salud: evidencia de efectos } & \text { https://www.redalyc.org/articulo.oa?id=1552 } \\ \text { umbral en el crecimiento económico. } & 66647006\end{array}$

\section{Ubicación de la revista}

Volumen número.

Lecturas de Economía núm. 94

\section{CUERPO}

\begin{tabular}{|c|c|c|c|c|}
\hline Metodología & Muestra & Instrumentos & Ética & Resultados \\
\hline Básico Descriptivo & 86 países & $\begin{array}{ll}\text { Ficha } & \text { de } \\
\text { recolección } & \text { de } \\
\text { datos Guía } & \text { de } \\
\text { análisis } & \\
\text { documental } & \end{array}$ & $\begin{array}{l}\text { Ética dt } \\
\text { investigación }\end{array}$ & $\begin{array}{l}\text { El estudio realizado muestra que } \\
\text { los } 86 \text { países presenta un mayor } \\
\text { nivel de educación esto tendría } \\
\text { efecto en el desempeño } \\
\text { económico determinando como } \\
\text { resultados ciudadanos saludables, } \\
\text { asegurando la productividad y el } \\
\text { desarrollo ciudadano. }\end{array}$ \\
\hline
\end{tabular}

\section{Conclusiones}

La calidad del gasto público aplicando políticas educativas con el objetivo de mejorar la asignación de recursos donde el conocimiento y el desempeño profesional sea productivo, lo que favorecería al desarrollo del ciudadano. 


\section{Tabla 05}

Datos de autor 05

\begin{tabular}{|c|c|c|c|c|c|}
\hline 5. & itor & Año & Título & Ubicación de la revista & Volumen y número \\
\hline $\begin{array}{l}\text { Willis, } \\
\text { Sean }\end{array}$ & Jason; Tanner, & 2020 & $\begin{array}{l}\text { Reducciones justas y equitativas a los } \\
\text { presupuestos estatales de educación: } \\
\text { evidencia y consideraciones para el año } \\
\text { fiscal } 2020 / 21 \text {. }\end{array}$ & $\begin{array}{l}\text { ht https://files.eric.ed.gov/fulltext/ED606130.pdf } \\
\text { Estados Unidos }\end{array}$ & $\begin{array}{l}\text { Perspectivas } \\
\text { Política: } \\
\text { No registra }\end{array}$ \\
\hline
\end{tabular}

\section{DESARROLLO}

\begin{tabular}{|c|c|c|c|c|c|}
\hline Metodología & Muestra & Instrumentos & $\begin{array}{l}\text { Ética de la } \\
\text { institución }\end{array}$ & Resultados & Conclusiones \\
\hline $\begin{array}{l}\text { Básico } \\
\text { Descriptivo }\end{array}$ & $\begin{array}{l}\text { Población } \\
\text { estudiantil de } \\
\text { Estados Unidos }\end{array}$ & $\begin{array}{l}\text { Revisión } \\
\text { Documental }\end{array}$ & Ética institucional & $\begin{array}{l}\text { Estados Unidos enfrenta una recesión } \\
\text { económica casi inevitable en los } \\
\text { próximos meses. Con una economía } \\
\text { en desaceleración vendrá una } \\
\text { contracción en los ingresos estatales, } \\
\text { incluido el financiamiento para la } \\
\text { educación pública. Dadas estas } \\
\text { realidades, ahora es un momento } \\
\text { oportuno de consideren las lecciones } \\
\text { aprendidas de ese período, así como } \\
\text { lo que dicen las investigaciones sobre } \\
\text { cómo el financiamiento y la } \\
\text { asignación de recursos afectan el } \\
\text { rendimiento académico de las } \\
\text { poblaciones estudiantiles más } \\
\text { vulnerables del país. }\end{array}$ & $\begin{array}{l}\text { Los líderes estatales deberían } \\
\text { considerar la adopción de un plan de } \\
\text { gastos que distribuya las reducciones } \\
\text { de manera algo uniforme en un plazo } \\
\text { de varios años. Esto proporcionaría un } \\
\text { horizonte para la planificación del } \\
\text { distrito escolar, lo que permitiría a los } \\
\text { líderes del distrito tomar decisiones } \\
\text { con una conciencia de los fondos que } \\
\text { probablemente estarán disponibles en } \\
\text { el futuro previsible. Los planificadores } \\
\text { estatales y locales también deben } \\
\text { considerar cómo se pueden usar los } \\
\text { fondos disponibles en conjunto con } \\
\text { otros recursos potenciales, como la } \\
\text { ayuda federal, para reducir el impacto } \\
\text { programático de la crisis } \\
\text { presupuestaria. }\end{array}$ \\
\hline
\end{tabular}

Análisis: El estudio muestra que los líderes estatales debe formular políticas enfocado a los presupuestos en educación para garantizar que la educación sea justo y equitativo. 


\section{Tabla 06}

Datos de investigación 06

\begin{tabular}{|c|c|c|c|c|}
\hline 6. Autor & Año & Título & Revista donde se ubica la Publicación & Volumen Y Numero \\
\hline Atchison, Drew & 2020 & $\begin{array}{l}\text { COVID-19 y la restricción de los } \\
\text { presupuestos estatales de educación: } \\
\text { implicaciones de equidad para el estado } \\
\text { de Nueva York. }\end{array}$ & $\begin{array}{l}\text { https://files.eric.ed.gov/fulltext/ED606245.pdf } \\
\text { Nueva York }\end{array}$ & $\begin{array}{l}\text { Institutos Americanos } \\
\text { de Investigación: } \\
\text { No registra }\end{array}$ \\
\hline
\end{tabular}

\section{DESARROLLO}

\begin{tabular}{|c|c|c|c|c|c|}
\hline Tipo de investigación & Muestra & Instrumentos & Ética & Resultados & Conclusiones \\
\hline Básico Descriptivo & $\begin{array}{l}\text { Alumnos } \\
\text { del estado } \\
\text { de New } \\
\text { York }\end{array}$ & $\begin{array}{l}\text { Revisión } \\
\text { Documental }\end{array}$ & $\begin{array}{l}\text { Ética } \\
\text { institucional }\end{array}$ & $\begin{array}{l}\text { El virus COVID-19, los estados están } \\
\text { comenzando a prepararse y comenzar } \\
\text { a abordar la contracción } \\
\text { presupuestaria que seguramente } \\
\text { vendrá sin dólares de estímulo } \\
\text { federal masivos. Dado que más del } \\
90 \% \text { de los ingresos de la educación } \\
\text { a nivel nacional provienen de fuentes } \\
\text { estatales y locales (Centro Nacional } \\
\text { de Estadísticas de Educación, 2020) } \\
\text { y aproximadamente el 20\% } \\
\text { representa la ejecucion presupuestal } \\
\text { en educación.. }\end{array}$ & $\begin{array}{l}\text { El efecto del COVID-19 en los } \\
\text { presupuestos de ayuda estatal del } \\
\text { distrito, se realizo una comparación de } \\
\text { las proyecciones del Presupuesto } \\
\text { Ejecutivo los cual nos da la mejor visión } \\
\text { de cómo los distritos probablemente } \\
\text { habrían sido financiados en ausencia de } \\
\text { la crisis de COVID-19. }\end{array}$ \\
\hline
\end{tabular}

Análisis: Los presupuestos en educación de Nueva York menciona que los distritos de mayor pobreza del estado son los más afectados por la crisis del COVID19.

Ciencia Latina Revista Científica Multidisciplinar, Ciudad de México, México.

ISN 2707-2207 / ISSN 2707-2215 (en línea), julio-agosto, 2021, Volumen 5, Número 4 https://doi.org/10.37811/cl_rcm.v5i4.769 p.6260 
Tabla 07

Datos de investigación 07

\begin{tabular}{lcllll}
\multicolumn{1}{c}{ 7. Autor } & Año & \multicolumn{1}{c}{ Nombre de la Investigación } & Revista donde se ubica la Publicación & Volumen Y Numero \\
\hline $\begin{array}{l}\text { Mishra, Karen E.; Mishra, Aneil } \\
\text { K. }\end{array}$ & $\begin{array}{l}\text { Innovaciones en la enseñanza de la } \\
\text { publicidad: enseñanza digital con un } \\
\text { presupuesto reducido. }\end{array}$ & $\begin{array}{l}\text { https://eric.ed.gov/?q=Budget+by+result+in+educ } \\
\text { ation\&pg=2\&id=EJ1251358 }\end{array}$ & $\begin{array}{l}\text { Revista de educación } \\
\text { publicitaria } \\
\text { vol 24, núm 1 }\end{array}$ \\
\hline
\end{tabular}

CUERPO

\begin{tabular}{|c|c|c|c|c|c|}
\hline Metodología & Muestra & Instrumentos & Ética & Resultados & Conclusiones \\
\hline Básico Descriptivo & Estudiantes & Cuestionario & Ética institucional & $\begin{array}{l}\text { Los estudiantes indicaron que Tool } \\
\text { Kit Fridays fue un método exitoso } \\
\text { para vincular la estrategia publicitaria } \\
\text { y las herramientas digitales } \\
\text { modernas. La Tabla } 4 \text { proporciona } \\
\text { los resultados de los comentarios de } \\
\text { los estudiantes recibidos al final de la } \\
\text { clase incluye los comentarios del } \\
73 \% \text { de los estudiantes al final del } \\
\text { semestre. Los que comentaron } \\
\text { detallaron cómo las sesiones del kit } \\
\text { de herramientas les ayudaron a } \\
\text { comprender mejor los principios } \\
\text { publicitarios aplicándolos a un } \\
\text { cliente real. }\end{array}$ & $\begin{array}{l}\text { El campo de la publicidad se está } \\
\text { integrando cada vez más con las } \\
\text { relaciones públicas y las redes } \\
\text { sociales (Kim, 2012). Como } \\
\text { resultado, muchos empleadores } \\
\text { esperan que los nuevos graduados } \\
\text { comprendan cómo navegar esto } \\
\text { desde una perspectiva integrada y } \\
\text { digital en sus nuevas carreras. El } \\
\text { desafío es que algunos cursos de } \\
\text { publicidad pueden impartirse desde } \\
\text { una perspectiva teórica y sin las } \\
\text { herramientas digitales que los } \\
\text { nuevos graduados necesitarán saber } \\
\text { utilizar. La innovación "Tool Kit } \\
\text { Friday" está diseñada para ayudar a } \\
\text { los estudiantes a aprender a usar } \\
\text { nuevas herramientas digitales y a } \\
\text { crear proyectos de clientes con esas } \\
\text { herramientas, al mismo tiempo que } \\
\text { aprenden estrategia publicitaria. }\end{array}$ \\
\hline
\end{tabular}




\section{Tabla 08}

Datos de investigación 08

8. Autor Año

Título

Link

Gordon, Nora; Loeb,

Susanna; Roza,

Marguerite; Taylor,

2020 Reducir los presupuestos distritales de manera responsable

https://files.eric.ed.gov/fulltext/ED607719.pdf

Proyecto

Investigación en

Eric

Taylor,

responsable

Educación para

la Recuperación núm. 4

\section{DESARROLLO}

\begin{tabular}{|c|c|c|c|c|c|}
\hline Metodología & Muestra & Instrumentos & Ética & Resultados & Conclusiones \\
\hline
\end{tabular}

Análisis. La investigación muestra los recortes del gasto escolar reduciendo el rendimiento en los estudiantes.

Ciencia Latina Revista Científica Multidisciplinar, Ciudad de México, México.

ISN 2707-2207 / ISSN 2707-2215 (en línea), julio-agosto, 2021, Volumen 5, Número 4 https://doi.org/10.37811/cl_rcm.v5i4.769 p.6262 
Tabla 09

Datos de investigación 09

\begin{tabular}{|c|c|c|c|c|c|}
\hline 9. Autor. & Año & Título & Ubicación de la revista & Volumen y núm & \\
\hline $\begin{array}{l}\text { YATUN, I. } \\
\text { MULYANI, S. } \\
\text { WINARNINGSIH, S. } 1 \\
\text { SUKMADILAGA, C. }\end{array}$ & 2021 & $\begin{array}{l}\text { El compromiso de los gobiernos } \\
\text { locales indonesios con la calidad } \\
\text { del sector educativo }\end{array}$ & $\begin{array}{l}\text { https://www.redalyc.org/jatsRepo/279/27966514029/ } \\
\text { 27966514029.pdf } \\
\text { Venezuela }\end{array}$ & $\begin{array}{l}\text { Utopía y } \\
\text { Latinoamericana: } \\
\text { vol. 26, núm.2 }\end{array}$ & Praxis \\
\hline
\end{tabular}

CUERPO

\begin{tabular}{|c|c|c|c|c|c|}
\hline Metodología & Muestra & Instrumentos & Ética & Resultados & Conclusiones \\
\hline $\begin{array}{l}\text { Básico } \\
\text { Descriptivo }\end{array}$ & $\begin{array}{l}267 \text { gobiernos } \\
\text { locales que } \\
\text { constan de } 63 \\
\text { gobiernos } \\
\text { municipales y } \\
204 \text { gobiernos } \\
\text { distritales }\end{array}$ & Cuestionario & $\begin{array}{l}\text { Ética de } \\
\text { investigación }\end{array}$ & $\begin{array}{l}\text { La influencia del compromiso de los jefes } \\
\text { regionales sobre la calidad del gasto del sector } \\
\text { educativo sobre la base del resultado de las } \\
\text { pruebas de hipótesis, el compromiso de los } \\
\text { responsables regionales tiene un efecto } \\
\text { positivo en la calidad del gasto del sector } \\
\text { educativo con un coeficiente de determinación } \\
\text { de } 0,299 \text {. La existencia de este efecto positivo } \\
\text { se debe a la autoridad regional de los jefes } \\
\text { sobre la planificación y aplicación de la APBD } \\
\text { de conformidad con las leyes. De acuerdo con } \\
\text { la Ley Número } 17 \text { de } 2003 \text { relativa a las } \\
\text { finanzas estatales, el Presidente delega las } \\
\text { facultades regionales de gestión financiera a } \\
\text { los jefes regionales para planificar, } \\
\text { presupuestar, implementar, administrar y } \\
\text { preparar un informe financiero como una } \\
\text { manifestación de su rendición de cuentas para } \\
\text { la ejecución del presupuesto regional. }\end{array}$ & $\begin{array}{l}\text { El resultado principal de este } \\
\text { estudio destaca la gran influencia } \\
\text { indirecta de la función de } \\
\text { supervisión del Plan de Desarrollo } \\
\text { de personas en el desempeño } \\
\text { educativo y la mejora de la función } \\
\text { de supervisión a lo largo del ciclo } \\
\text { presupuestario añade valor a la } \\
\text { calidad del gasto del sector } \\
\text { educativo y puede fomentar } \\
\text { simultáneamente un mejor } \\
\text { desempeño educativo. Este estudio } \\
\text { también encontró una relación } \\
\text { positiva entre el compromiso de los } \\
\text { responsables regionales y el } \\
\text { desempeño educativo, tanto directa } \\
\text { como indirectamente, a través de la } \\
\text { calidad del gasto del sector } \\
\text { educativo }\end{array}$ \\
\hline
\end{tabular}

Análisis: El estudio tiene como objetivo mejorar la calidad del gasto público en el sector educación y mejorar el rendimiento educativo. 


\section{Tabla 10}

Datos de investigación 10

\begin{tabular}{|c|c|c|c|c|}
\hline 10. Autor & A ño & Título & Link & Revista \\
\hline $\begin{array}{l}\text { Tomás Darío Marín- } \\
\text { Velásquez, Dany Day } \\
\text { Josefina Arriojas- } \\
\text { Tocuyo, Erika Raquel } \\
\text { Acosta-Roa }\end{array}$ & 2020 & $\begin{array}{l}\text { Gasto público y familiar en educación en Perú: } \\
\text { comparativa con Sudamérica }\end{array}$ & $\begin{array}{l}\text { file:///D:/DESCARGAS/Gastopublicoeducacio } \\
\text { n\%20(2).pdf } \\
\text { Perú }\end{array}$ & $\begin{array}{l}\text { Gasto público } \\
\text { y familiar en } \\
\text { educación en } \\
\text { Perú } \\
\text { vol. } 8 \text {, núm. } 15\end{array}$ \\
\hline
\end{tabular}

\section{DESARROLLO}

\begin{tabular}{|c|c|c|c|c|c|}
\hline Metodología & Muestra & Instrumentos & Ética & Resultados & Conclusiones \\
\hline $\begin{array}{l}\text { Básico } \\
\text { Descriptivo } \\
\text { comparativo }\end{array}$ & $\begin{array}{l}\text { Hogares en } \\
\text { educación } \\
\text { - de los años } \\
2000 \text { y } 2015 \\
\text { del Perú, }\end{array}$ & $\begin{array}{l}\text { Cuestionario - } \\
\text { Ficha de } \\
\text { observación }\end{array}$ & $\begin{array}{l}\text { Código de } \\
\text { ética } \\
\text { instituciona } \\
1\end{array}$ & $\begin{array}{l}\text { La ejecución del gasto en educación en } \\
\text { el Perú es más baja en el mundo y se } \\
\text { observa que va en aumento, no se tiene } \\
\text { eficiencia, lo que evidencia } \\
\text { desigualdades en educación de calidad, } \\
\text { reduciendo la tasa de matrícula y el } \\
\text { retraso escolar que produce repitencia y } \\
\text { deserción. }\end{array}$ & $\begin{array}{l}\text { La educación en el Perú presenta un incremento } \\
\text { equivalente al de Argentina, Brasil y Colombia, } \\
\text { menor al de Bolivia y Paraguay y mayor al de } \\
\text { Ecuador y Uruguay. Según lo analizado, el gasto } \\
\text { público en educación no garantiza la calidad de la } \\
\text { misma. }\end{array}$ \\
\hline
\end{tabular}

Análisis. En la investigación se estudia el gasto público de los hogares en educación en Perú no garantiza la calidad en los aprendizajes. 
Tabla 11

Datos de investigación 11

\begin{tabular}{|c|c|c|c|c|}
\hline 11. Autor & Año & Título & Link & Revista \\
\hline $\begin{array}{l}\text { Rebecca Morris } \\
\text { Graeme Dobson }\end{array}$ & 2020 & $\begin{array}{l}\text { Gasto de la prima por alumno: ¿qué influye en la toma } \\
\text { de decisiones de los líderes? }\end{array}$ & $\begin{array}{l}\text { https://journals.sagepub.com/doi/pdf/10.1177/17 } \\
41143220905062\end{array}$ & $\begin{array}{l}\text { Gestión } \\
\text { educativa } \\
\text { Administració } \\
\text { n y liderazgo } \\
\text { vol 49, núm. } 2\end{array}$ \\
\hline
\end{tabular}

DESARROLLO

\begin{tabular}{|c|c|c|c|c|c|}
\hline Metodología & Muestra & Instrumentos & Ética & Resultados & Conclusiones \\
\hline $\begin{array}{l}\text { Básico } \\
\text { Descriptivo }\end{array}$ & $\begin{array}{l}21 \quad \text { líderes } \\
\text { escolares }\end{array}$ & $\begin{array}{l}\text { Ficha de } \\
\text { recolección de } \\
\text { datos } \\
\text { entrevistas }\end{array}$ & $\begin{array}{l}\text { Código de } \\
\text { ética } \\
\text { instituciona } \\
1\end{array}$ & $\begin{array}{l}\text { Las perspectivas detalladas sobre estas } \\
\text { cuestiones, y para entender las } \\
\text { complejidades potenciales que implica } \\
\text { la toma de decisiones financieras, } \\
\text { realizamos una serie de entrevistas en } \\
\text { profundidad y semiestructuradas con } 21 \\
\text { líderes escolares, gobernadores y } \\
\text { representantes de las autoridades locales } \\
\text { responsables del gasto del PP. } \\
\text { Reconociendo que la responsabilidad } \\
\text { del gasto del PP está organizada y } \\
\text { delegada de diferentes maneras en todos } \\
\text { los colegios, buscamos captar } \\
\text { variaciones en relación con los roles y } \\
\text { contextos de los líderes escolares } \\
\text { involucrados. }\end{array}$ & $\begin{array}{l}\text { Las instituciones educativas juegan un rol } \\
\text { importante en desarrollo de los niños, pero } \\
\text { incluso con recursos adicionales es poco } \\
\text { probable que puedan compensar las } \\
\text { desigualdades que los niños experimentan. Por } \\
\text { lo tanto, desarrollar e invertir en políticas que } \\
\text { busquen reducir la pobreza, y que aborden y } \\
\text { mejoren las intersecciones domésticas, } \\
\text { comunitarias y escolares asociadas con } \\
\text { desventajas y logros insuficientes, es probable } \\
\text { que sea un enfoque importante y beneficioso. }\end{array}$ \\
\hline
\end{tabular}

Ciencia Latina Revista Científica Multidisciplinar, Ciudad de México, México.

ISN 2707-2207 / ISSN 2707-2215 (en línea), julio-agosto, 2021, Volumen 5, Número 4.

https://doi.org/10.37811/cl_rcm.v5i4.769 p.6265 
Tabla 12

Datos de investigación 12

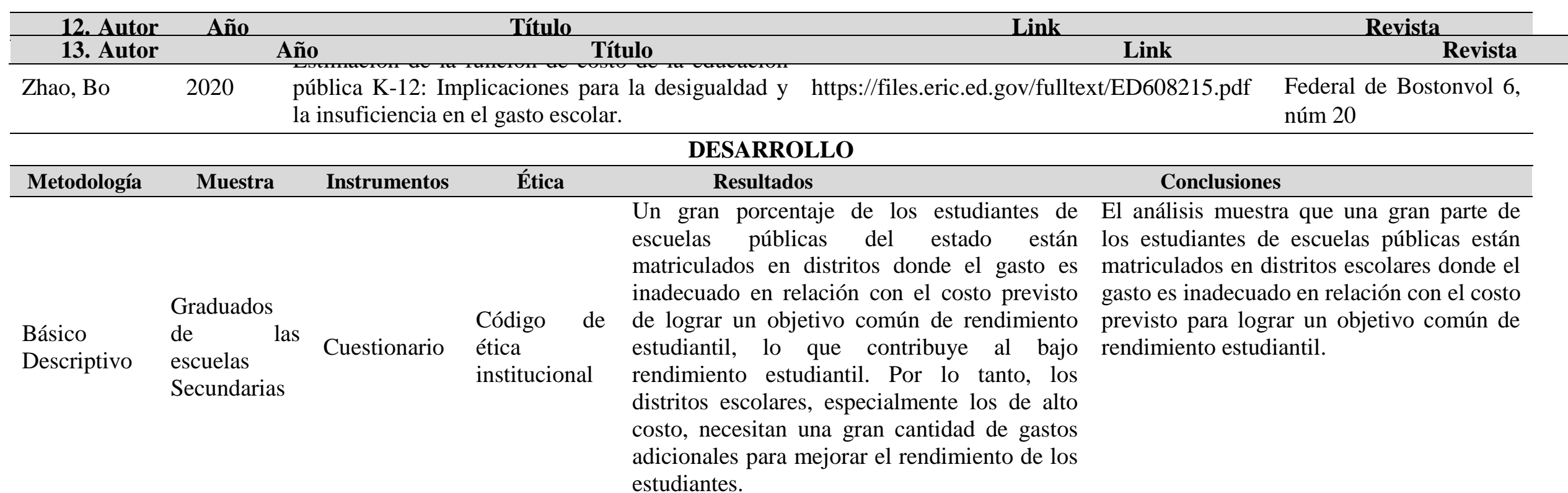

Análisis. El estudio muestra que las instituciones educativas tienen el costo alto y el gasto menos ejecutado para lograr el objetivo de rendimiento de los estudiantes. 
Hahn, Heather; Lou,

Cary; Isaacs, Julia B

; Lauderback,

Eleanor; Daly,

Hannah; Steuerle,

Eugene
C.
Compartir para niños 2020: Informe sobre los gastos https://files.eric.ed.gov/fulltext/ED607695.pdf Instituto Urbano

federales en niños hasta 2019 y proyecciones futuras

\section{DESARROLLO}

\begin{tabular}{|c|c|c|c|c|c|}
\hline Metodología & Muestra & Instrumentos & Ética & Resultados & Conclusiones \\
\hline $\begin{array}{l}\text { Básico } \\
\text { Descriptivo }\end{array}$ & Niños & $\begin{array}{l}\text { Ficha de } \\
\text { recolección de } \\
\text { datos }\end{array}$ & $\begin{array}{l}\text { Código de } \\
\text { ética } \\
\text { instituciona } \\
1\end{array}$ & $\begin{array}{l}\text { Las proyecciones presupuestarias se } \\
\text { basan en la referencia de marzo de } 2020 \text {, } \\
\text { refleja el pronóstico económico hace } 10 \\
\text { años en gran medida son inciertas } \\
\text { porque no se anticiparon a los efectos de } \\
\text { la pandemia COVID-19. }\end{array}$ & $\begin{array}{l}\text { Los gastos públicos dirigidos a los niños pueden } \\
\text { ayudar a garantizar y alcanzar su máximo } \\
\text { potencial. Aunque los padres y las familias } \\
\text { proporcionan la mayoría de las necesidades } \\
\text { básicas de los niños, la sociedad en general } \\
\text { desempeña un papel vital en el apoyo a su } \\
\text { desarrollo saludable. Por ejemplo, los beneficios } \\
\text { nutricionales, la asistencia para la vivienda y los } \\
\text { programas de seguro de salud apoyan las } \\
\text { necesidades de los niños de alimentos, vivienda y } \\
\text { buena salud, mientras que las inversiones en } \\
\text { educación temprana y escuelas públicas } \\
\text { promueven el aprendizaje y la igualdad de } \\
\text { oportunidades. }\end{array}$ \\
\hline
\end{tabular}
oportunidades.

Análisis. La investigación ayuda a garantizar que le gasto público estén dirigidos a los niños y garantizar el máximo potencial en la educación temprana y la igual de oportunidades

\section{Tabla 13}

Datos de investigación 13

\section{Tabla 14}

Datos de investigación 14 


\begin{tabular}{|c|c|c|c|c|}
\hline 14. Autor & Año & Título & Link & Revista \\
\hline
\end{tabular}

\section{DESARROLLO}

\begin{tabular}{|c|c|c|c|c|c|}
\hline Metodología & Muestra & Instrumentos & Ética & Resultados & Conclusiones \\
\hline $\begin{array}{l}\text { Básico } \\
\text { Descriptivo }\end{array}$ & $\begin{array}{l}\text { Países como } \\
\text { Unión } \\
\text { Europea } \\
\text { (Brasil, } \\
\text { Rusia, India, } \\
\text { China y } \\
\text { Sudáfrica) }\end{array}$ & $\begin{array}{l}\text { Revisión } \\
\text { Documental }\end{array}$ & $\begin{array}{l}\text { Código de } \\
\text { ética } \\
\text { institucional }\end{array}$ & $\begin{array}{l}\text { De acuerdo con los objetivos de la } \\
\text { educación establecidos por la Unión } \\
\text { Europea y los países BRICS, la } \\
\text { educación tiene como objetivo: } \\
\text { fortalecer la educación y la formación } \\
\text { profesional, fomentar la educación de } \\
\text { calidad y el desempeño profesional } \\
\text { del personal: al observar estos tres } \\
\text { objetivos, es evidente que el objetivo } \\
\text { de la educación es producir } \\
\text { trabajadores calificados que puedan } \\
\text { unirse a la fuerza laboral de un país y } \\
\text { promover aún más la educación. }\end{array}$ & $\begin{array}{l}\text { El gasto en educación tiene una tasa de } \\
\text { crecimiento media de } 19 \text { mil millones de dólares } \\
\text { por cada } 1 \% \text { invertido en educación. La diferencia } \\
\text { en la tasa de rendimiento del (PBI) entre la Unión } \\
\text { Europea y los BRICS es principalmente, pero no } \\
\text { se limita a, la atribución del coeficiente de error, } \\
\text { ya que deben tenerse en cuenta factores como la } \\
\text { migración, la administración y la población. }\end{array}$ \\
\hline
\end{tabular}

\section{Tabla 15}

Datos de investigación 15 


\begin{tabular}{|c|c|c|c|c|}
\hline 15. Autor & Año & Título & Link & Revista \\
\hline
\end{tabular}

\section{DESARROLLO}

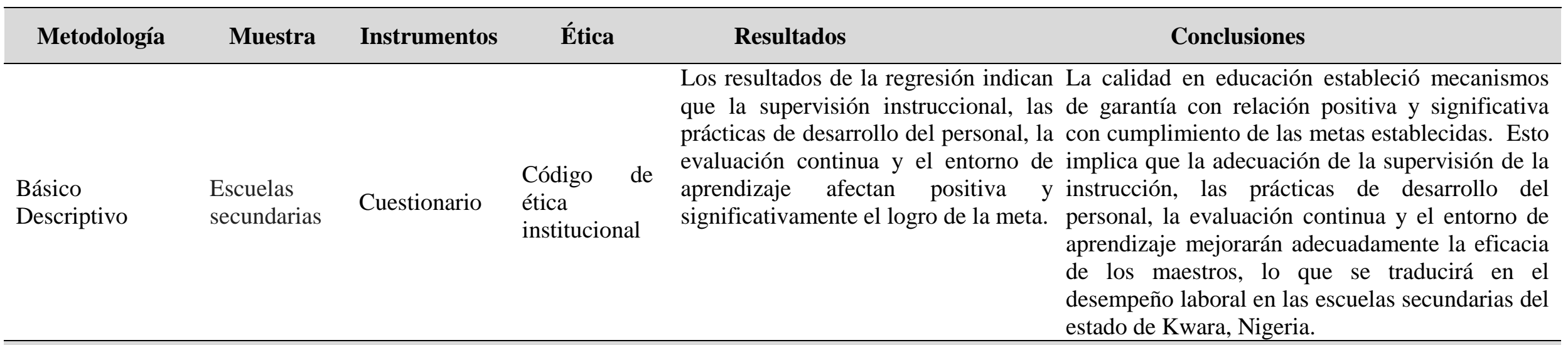

Análisis. La educación es considera como un mecanismo para el desarrollo eficaz y el cumplimiento de las metas establecidas. 


\section{Tabla 16}

Datos de investigación 16

\begin{tabular}{|c|c|c|c|c|}
\hline 16. Autor & Ã̃o & Título & Link & Revista \\
\hline $\begin{array}{l}\text { Yong, } \\
\text { Mengshu; Zhang, } \\
\text { Yuxin }\end{array}$ & 2021 & $\begin{array}{l}\text { Alivio de la pobreza mediante la educación: exploración } \\
\text { y experiencia de China - Análisis basado en la política de } \\
\text { alivio de la pobreza mediante la educación de China }\end{array}$ & $\begin{array}{l}\text { https://files.eric.ed.gov/fulltext/EJ1286819.pdf } \\
\text { China }\end{array}$ & $\begin{array}{l}\text { Perspectivas } \\
\text { científicas } \\
\text { Fronteras de } \\
\text { la educación } \\
\text { vol. } 8 \text {, núm.1 }\end{array}$ \\
\hline
\end{tabular}

\section{DESARROLLO}

\begin{tabular}{|c|c|c|c|c|c|}
\hline Metodología & Muestra & Instrumentos & Ética & Resultados & Conclusiones \\
\hline
\end{tabular}

Análisis. El estudio muestra que la educación ha cumplido un rol importante en la promoción del desarrollo económico. 
Tabla 17

Datos de investigación 17

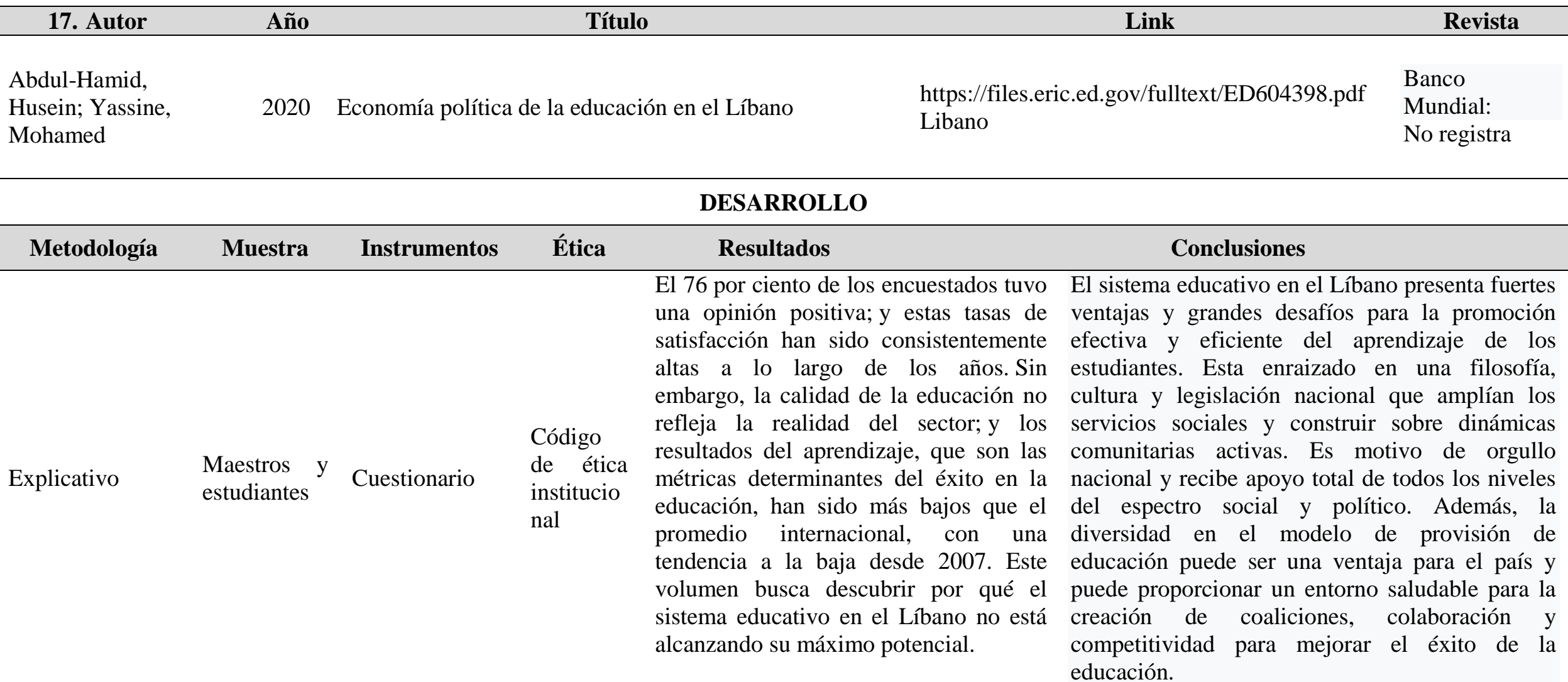

Análisis. La educación proporciona un entorno saludable para la creación de coaliciones, colaboración, competitividad para alcanzar su máximo potencial. 


\section{Tabla 18}

Datos de investigación 18

\begin{tabular}{|c|c|c|c|c|}
\hline 18. Autor & Año & Título & Link & Revista \\
\hline Tanjung, Ellisa Fitri & 2020 & $\begin{array}{l}\text { Impacto del bienestar público, la competitividad y la eficacia } \\
\text { del gobierno en la calidad de la educación en los países } \\
\text { asiáticos }\end{array}$ & $\begin{array}{l}\text { https://files.eric.ed.gov/fulltext/EJ1284 } \\
\text { 640.pdf }\end{array}$ & $\begin{array}{l}\text { Revista } \\
\text { chipriota de } \\
\text { ciencias de la } \\
\text { educación } \\
\text { vol.15, núm.6 }\end{array}$ \\
\hline
\end{tabular}

\section{DESARROLLO}

\begin{tabular}{|c|c|c|c|c|c|}
\hline Metodología & Muestra & Instrumentos & Ética & Resultado: & Conclusiones \\
\hline $\begin{array}{l}\text { Básico } \\
\text { Descriptivo }\end{array}$ & $\begin{array}{l}10 \text { países } \\
\text { asiáticos } \\
\text { (Indonesia, } \\
\text { India, } \\
\text { Tailandia, } \\
\text { Corea del } \\
\text { Sur, Sri } \\
\text { Lanka, } \\
\text { Vietnam, } \\
\text { Malasia, } \\
\text { Japón, China } \\
\text { y Bahrein) }\end{array}$ & Cuestionario & $\begin{array}{l}\text { Código de } \\
\text { ética } \\
\text { institucional }\end{array}$ & $\begin{array}{l}\text { En la investigación se recopilo durante } 25 \\
\text { años para } 10 \text { países asiático incluido la } \\
\text { muestra del bienestar público, la eficacia } \\
\text { del Gobierno, la tasa de alfabetización y el } \\
\text { crecimiento de la población repercuten } \\
\text { significativamente en los logros de } \\
\text { aprendizaje. }\end{array}$ & $\begin{array}{l}\text { La calidad de la educación es el } \\
\text { componente vital para el crecimiento del } \\
\text { país, ya que determina la competitividad } \\
\text { de sus ciudadanos y aumenta las } \\
\text { posibilidades de desarrollo de un país. La } \\
\text { calidad educativa en la región de Asia } \\
\text { incorporo el bienestar público, la eficacia y } \\
\text { el nivel de competitividad del Gobierno, la } \\
\text { tasa de alfabetización y el desarrollo de la } \\
\text { población. Las políticas mejoraran los } \\
\text { aprendizajes en los países analizados con } \\
\text { la participación de ciudadanos. El estudio } \\
\text { ayudará a los futuros investigadores a } \\
\text { comprender los sistemas de gobernanza de } \\
\text { manera efectiva para la educación. }\end{array}$ \\
\hline
\end{tabular}

Análisis. El estudio muestra que la calidad de la educación es el componente vital para el crecimiento del país, ya que determina la competitividad de sus ciudadanos y aumenta las posibilidades de desarrollo de un país. 
Tabla 19

Datos de investigación 19

\begin{tabular}{|c|c|c|c|c|}
\hline 19. Autor & Año & Título & Link & Revista \\
\hline $\begin{array}{l}\text { Tabatadze, } \\
\text { Shalva; Chachkhiani, } \\
\text { Ketevan }\end{array}$ & 2021 & $\begin{array}{l}\text { COVID-19 y la enseñanza remota de emergencia en el } \\
\text { país de Georgia: ¿catalizador del cambio y las reformas } \\
\text { educativas en Georgia? }\end{array}$ & $\begin{array}{l}\text { https://eric.ed.gov/?q=Budget+management } \\
\text { +by+result+and+quality+of+public+spendi } \\
\text { ng+on+education\&ff1=dtyIn_2021\&pg=2 } \\
\text { \&id=EJ1285261 }\end{array}$ & $\begin{array}{l}\text { Revista de la } \\
\text { Asociación } \\
\text { Estadounidense } \\
\text { de Estudios } \\
\text { Educativos: } \\
\text { vol. 57, núm. } 1\end{array}$ \\
\hline
\end{tabular}

\section{DESARROLLO}

\begin{tabular}{|c|c|c|c|c|c|}
\hline Metodología & Muestra & Instrumentos & Ética & Resultados & Conclusiones \\
\hline $\begin{array}{l}\text { Diseño } \\
\text { propositivo }\end{array}$ & 29 escuelas & $\begin{array}{l}\text { Ficha de } \\
\text { recopilación } \\
\text { de datos, } \\
\text { entrevistas }\end{array}$ & $\begin{array}{l}\text { Código de } \\
\text { ética } \\
\text { institucional }\end{array}$ & $\begin{array}{l}\text { El estudio de investigación reveló } \\
\text { desafíos, así como desarrollos } \\
\text { positivos experimentados por las } \\
\text { escuelas de idiomas no georgianos } \\
\text { durante la enseñanza remota de } \\
\text { emergencia. Como se mencionó } \\
\text { anteriormente, estos hallazgos y } \\
\text { análisis se basan en los siguientes } \\
\text { cuatro dominios: disponibilidad de } \\
\text { recursos; preparación de las escuelas, } \\
\text { implicaciones para el sistema } \\
\text { educativo; impacto de la enseñanza } \\
\text { remota de emergencia. }\end{array}$ & $\begin{array}{l}\text { Los hallazgos de este estudio tienen fuertes } \\
\text { implicaciones para las políticas educativas de } \\
\text { Georgia en el futuro. Si estas oportunidades y } \\
\text { desafíos revelados durante la pandemia de } \\
\text { COVID-19 desencadenan reformas educativas o } \\
\text { no, depende de andtiples } \\
\text { factores. Afortunadamente, el Gobierno de } \\
\text { Georgia se ha declarado dispuesto a implementar } \\
\text { estas reformas. }\end{array}$ \\
\hline
\end{tabular}

Análisis. El estudio de investigación reveló desafíos, así como desarrollos positivos experimentados por las escuelas de idiomas no georgianos durante la enseñanza remota de emergencia. 
Tabla 20

Datos de investigación 20

\begin{tabular}{|c|c|c|c|c|}
\hline 20. Autor & Año & Título & Link & Revista \\
\hline $\begin{array}{c}\text { Ozkayran, Secil } \\
\text { Eda; Yetis Abali, } \\
\text { Aslihan; Abali, Ahmet }\end{array}$ & 2020 & $\begin{array}{l}\text { Las opiniones de los docentes sobre la gestión de } \\
\text { crisis en los servicios de orientación. }\end{array}$ & https://files.eric.ed.gov/fulltext/EJ1284809.pdf & $\begin{array}{l}\text { Proceso } \\
\text { educativo: } \\
\text { Revista } \\
\text { internacional } \\
\text { vol.9, núm. } 4\end{array}$ \\
\hline
\end{tabular}

\section{DESARROLLO}

\begin{tabular}{|c|c|c|c|c|c|}
\hline Metodología & Muestra & Instrumentos & Ética & Resultados & Conclusiones \\
\hline $\begin{array}{c}\text { Básico } \\
\text { Descriptivo }\end{array}$ & 25 Maestros & $\begin{array}{l}\text { Encuestas y } \\
\text { Formulario de } \\
\text { entrevistas }\end{array}$ & $\begin{array}{l}\text { Código de } \\
\text { ética } \\
\text { institucional }\end{array}$ & $\begin{array}{l}\text { No hubo efectos directos de la calidad } \\
\text { preescolar según la medición de la } \\
\text { puntuación de interacción ECERS-R } \\
\text { en ninguna de las variables de } \\
\text { resultado, pero hubo efectos indirectos } \\
\text { significativos de la calidad preescolar } \\
\text { a través de la persistencia en el logro } \\
\text { de las matemáticas (b }=.04, \text { IC del } \\
95 \% \text { [0,008, 0,073], SE }=.017, \mathrm{p}= \\
.015) \text { y el logro de lectura }(\mathrm{b}=.04, \mathrm{IC} \\
\text { del 95\% [0,002, } 0,086], \mathrm{SE}=.022, \mathrm{p}= \\
.041) \text {. Por lo tanto, la persistencia de } \\
\text { los niños en la primera. }\end{array}$ & $\begin{array}{l}\text { Los hallazgos de este estudio apoyan la } \\
\text { posibilidad de que la capacidad de los niños para } \\
\text { persistir en situaciones de aprendizaje pueda ser } \\
\text { atacada directamente por los esfuerzos de } \\
\text { intervención temprana. Mejorar el desempeño y } \\
\text { habilidades que los niños persevere en tareas } \\
\text { difíciles es crucial, ya que representan } \\
\text { características de comportamiento clave que son } \\
\text { relevantes para el éxito en la escuela, la } \\
\text { educación superior y el lugar de trabajo en la } \\
\text { edad adulta. }\end{array}$ \\
\hline
\end{tabular}

Análisis. La investigación apoyan a la capacidad de los niños para persistir en situaciones de aprendizaje pueda ser atacada directamente por los esfuerzos de intervención temprana.

Ciencia Latina Revista Científica Multidisciplinar, Ciudad de México, México.

ISN 2707-2207 / ISSN 2707-2215 (en línea), julio-agosto, 2021, Volumen 5, Número 4 https://doi.org/10.37811/cl_rcm.v5i4.769 p.6274 


\section{DISCUSIÓN}

La intervención del gobierno en el presupuesto público debería responder a las necesidades de las políticas públicas implementadas. La calidad de la educación es el componente vital para el crecimiento del país, ya que determina la competitividad de sus ciudadanos y aumenta las posibilidades de desarrollo de un país (Tanjung , 2020).

Asimismo, las escuelas representan una labor primordial en la vida de los niños, pero incluso con recursos adicionales es poco probable que puedan compensar las desigualdades que los niños experimentan en las escuelas. Desarrollar e invertir en políticas que busquen reducir la pobreza, y que aborden y mejoren las intersecciones domésticas, comunitarias y escolares asociadas con desventajas y logros insuficientes, es probable que sea un enfoque importante y beneficioso (Morris \& Dobson, 2020).

Sin embargo, la educación en las escuelas pública es fundamental para mejorar los resultados del desarrollo infantil y garantizar que las próximas generaciones de niños estén mejor preparadas para la escuela. (Dev Bhatta, Rahman, Rahman , Sharma, \& Adms , 2020).

Además, Los gastos públicos dirigidos a los niños pueden ayudar a garantizar que los niños reciban los recursos que requieren para lograr su enorme potencial. Aunque los padres y las familias proporcionan la mayoría de las necesidades básicas de los niños, la sociedad en general desempeña un papel vital en el apoyo a su desarrollo saludable. Por ejemplo, los beneficios nutricionales, la asistencia para la vivienda y los programas de seguro de salud apoyan las necesidades de los niños de alimentos, vivienda y buena salud, mientras que las inversiones en educación temprana y escuelas públicas promueven el aprendizaje y la igualdad de oportunidades (Hahn, y otros, 2020).

Justamente, se requiere relacionar los recursos del presupuesto con los resultados logrados, tanto en resultados intermedios (cobertura) como finales (competencia suficiente del alumno), Un gran porcentaje de los estudiantes de escuelas públicas del estado están matriculados en distritos donde el gasto es inadecuado en relación con el costo previsto de lograr un objetivo común de rendimiento estudiantil, lo que contribuye al bajo rendimiento (Zhao,2020).

Sin embargo, con una economía en desaceleración vendrá una contracción en los ingresos estatales, incluido el financiamiento para la educación pública. Dadas estas realidades, ahora es un momento oportuno de formular políticas consideren el 
financiamiento y la asignación de recursos a las poblaciones estudiantiles más vulnerables del país (Willis \& Tanner, 2020).

Por otro lado, el compromiso de los jefes regionales sobre la calidad del gasto público del sector educativo tiene un efecto positivo sobre la planificación y aplicación del informe financiero como una manifestación de su rendición de cuentas para la ejecución del presupuesto regional (Yatun, Mulyani, Winarningsih, \& Sukmadilaga, 2021).

Finalmente, en el tiempo de elaborar el presupuesto se dispone con muchas limitaciones para confrontar el retraso de los procedimientos. La ausencia de un mecanismo de planificación no permite priorizar y hacer uso de manera racional los recursos financieros que solucione a las necesidades de la población

\section{CONCLUSIONES}

Los estudios de mayor predominancia en la revisión sistemática son los básicos, con diseño descriptivo; a ello se adiciona los estudios comparativos y propositivos.

La revisión sistemática, se sustentó en evidencia de la gestión del presupuesto por resultado, determinante en la calidad del gasto y el uso eficiente del presupuesto público con objetivos educativos que demanda la población.

La gestión del presupuesto por resultado promueve una gestión educativa eficiente y descentralizada con enfoque participativo de la sociedad, familias, ciudadanos y ciudadanas para la implementación de políticas educativas basadas en la gestión por resultados con enfoque a la comunidad de aprendizaje profesional y a la prestación de servicios educativos. Como el desarrollo profesional del maestro, el fortalecimiento de mecanismos de garantía que asegure la cantidad suficiente de materiales educativos.

La calidad del gasto en educación no garantiza que los niños reciban los recursos que demandan para obtener la calidad de los aprendizajes ya que ellos necesitan alimentos, vivienda y buena salud. El sector educativo señala que la práctica educativa añade valor a la eficiencia del gasto público y promueve las inversiones en las escuelas públicas, el desempeño educativo juega una labor importante en el desarrollo de los niños originando la igualdad de oportunidades de aprendizaje a largo del ciclo presupuestario.

Asimismo, La gestión del presupuesto por resultado permite mejorar las condiciones institucionales, principalmente incorporando el bienestar público, la eficacia y competitividad del gobierno, en las políticas educativas. La calidad del gasto público optimiza y da solución a problemática existente. 
Finalmente, se recomienda formular estrategias basadas en técnicas de elaboración de la gestión del presupuesto que permite mejorar el presupuesto existente y manejarlo adecuadamente en un eficiente gasto que esté de acuerdo a lo planificado evitando las recargadas de comisiones de compras o hasta la corrupción de trabajadores.

\section{REFERENCIAS BIBLIOGRAFICAS}

Abdul, H. H., \& Yassine, M. (2020). Economía política de la Educación en el Líbano. Banco Mundial. Obtenido de https://files.eric.ed.gov/fulltext/ED604398.pdf

Atchison, D. (Mayo de 2020). COVID -19 y la restricción de los prespuesto estatales de educación: implicaciones de equidad para el estado de Nueva York. Instituto Americanos de Investigación, 1-47. Obtenido de https://files.eric.ed.gov/fulltext/ED606245.pdf

Batista Rodríguez , L. F., Leyva Figueredo , P. A., \& Mendoza Tauler , L. L. (2021). Gestión y desarrollo de la formación laboral en la educación actual. Ciencias Técnicas, 27(1), 28-37. Obtenido de https://www.redalyc.org/jatsRepo/1815/181565709003/html/index.html

Bouchamma, Y., \& April, D. (2020). La comunidad de aprendizaje para implementar el enfoque de gestión basa en resultados (GBR) en Quebec. Revista Canadiense de Administración Publica, 192, 77-85. Obtenido de https://files.eric.ed.gov/fulltext/EJ1252284.pdf

Dev Bhatta, S., Rahman, T., Rahman , N., Sharma, U., \& Adms , L. (2020). El panomarama de la educacion infantil en Bangladesh. Banco mundial, 117. Obtenido de https://eric.ed.gov/?q=Bangladesh+The+Landscape+of+Early+Childhood+Educati on+in+Bangladesh\&id=ED604387

Gordon , N., Loeb, S., Roza, M., \& Taylor , E. (Julio de 2020). Reducir los presupuestos distritales de manera responsable. Proyecto de Investigación en Educación para la Recuperación, 4, 1-6. Obtenido de https://files.eric.ed.gov/fulltext/ED607719.pdf

Hahn , H., Lou, C., Isaacs, J. B., Lauderback, E., Hannah, D., \& Steurele, C. E. (Julio de 2020). Compartir para niños 2020: Informe sobre los gastos federales en niños hasta 2019 y proyecciones futuras. Instituto Urbano, 1-64. Obtenido de https://files.eric.ed.gov/fulltext/ED607695.pdf

Hernández, R. (2014). Metodología de la Investigación (Sexta ed.). México: Mc Graw Hill. 
Marin , T. D., Arriojas tocuyo, D. D., \& Acosta Roa, E. R. (Julio de 2020). Gasto público y familiar en educación en Peru: comparativa con Sudamérica. Revista electronica de Investigación en Ciencias Economicas Abriendo Camino al conocimiento, 8(15), 39-35. doi:DOI: 10.5377/reice.v8i15.9944

Mishra, K. E., \& Mishra, A. K. (mayo de 2020). Innovaciones en la enseñanza de la publicidad: enseñanza digital con un presupuesto reducido. Revista de educación publicitaria, 24(1), 8-20. doi:http://dx.doi.org/10.1177/1098048220913342

Monterubbianesi, P. D., Rojas, M. L., \& Dabús, C. D. (Enero-Junio de 2021). Educación y salud: evidencia de efectos umbral en el crecimiento económico. Lecturas de Economia(94), 195-231. doi:DOI: https://doi.org/10.17533/udea.le.n94a342459

Morris, R., \& Dobson, G. (Marzo de 2020). Gasto de la prima por alumno: ¿qué influye en la toma de decisiones de los lideres? Gestión educativa administrativa y liderazgo, 49(2), 284-302. doi:https://doi.org/10.1177/1741143220905062

Otto, M. (2020). El conocimiento como moneda: una exploración comparativa de la relación entre gasto en educación y el producto interno bruto de la Unión Europea y los paises BRICS. Sociedad Bulgara de Educación comparada, 18. Obtenido de https://files.eric.ed.gov/fulltext/ED608413.pdf

Ozkayran, S. E., Abali, A. Y., \& Abali, A. (2020). Las oponiones de los docentes sobre la gestión de crisis en los servicios de orientación. Proceso educativo: Revista internacional, 9(4). doi:http://dx.doi.org/10.22521/edupij.2020.94.2

Sanders, R., \& Zhang, S. (2020). Utilización eficaz de los recursos en las escuelas públicas de Arkansas. Revista Internacional de Gestion Educativa, 35(1). doi:http://dx.doi.org/10.1108/IJEM-05-2019-0163

Sayuti , O. A., Adetiba, A. A., Tukur, M. A., \& Abubakar, L. A. (Febrero de 2020). Mecanismos de garantía de calidad y educación básica universal en las escuelas secundarias públicas universal en las escuelas secundarias públicas del estado de Kwara, Nigeria. Revista de Educación y aprendizaje, 14(1), 1-7. doi:10.11591

Tabatadze, S., \& Chachkhiani, K. (2021). COVID-19 y la enseñanza remota de emergencia en el país de Georgia: ¿catalizador del cambio y las reformas educativas en Georgia? Revista de la Asociación Estadounidense de Estudios Educativos, 57(1), 78-95. doi:http://dx.doi.org/10.1080/00131946.2020.1863806 
Tanjung, E. F. (2020). Impacto del bienestar público, la competitividad y el gobierno en la calidad de la educación en los países asiáticos. Revista chipriota de ciencias de la educación, 15(6). Obtenido de https://files.eric.ed.gov/fulltext/EJ1284640.pdf

Willis, J., \& Tanner, S. (2020). Reduciones justas y equitativas a los presupuestos estatales de educación: evidencias y consideraciones para el año fiscal 2020/21. Perspectiva de Politica, 1-6. Obtenido de https://files.eric.ed.gov/fulltext/ED606130.pdf

Yatun, I., Mulyani, S., Winarningsih, s., \& Sukmadilaga, C. (2021). El compromiso de los gobiernos loocales indonesios con la calidad del sector educativo. UTOPÍA Y PRAXIS LATINOAMERICANA, doi:http://doi.org/10.5281/zenodo.4678915

Yong, M., \& Zhang, Y. (2021). Alivio de la pobreza mediante la educación: exploración y expereiencia de China - Análisis basado en la política de alivio de la pobreza mediante la educación de china. Perspectiva científicas Fronteras de la educación, 8(1), 959-973. doi:Doi: 10.15354

Zhao, B. (Marzo de 2020). Estimación de la función de costo de la educación pública K12: Implicaciones para la desigualdad y la insuficiencia en el gasto escolar. 6(20). doi:https://doi.org/10.29412/res.wp.2020.06 